\title{
LXXIV. The sum of an infinite series as the solution of a linear differential equation
}

\section{I.J. Schwatt}

To cite this article: I.J. Schwatt (1914) LXXIV. The sum of an infinite series as the solution of a linear differential equation, Philosophical Magazine Series 6, 27:160, 659-662, DOI: $10.1080 / 14786440408635135$

To link to this article: http://dx.doi.org/10.1080/14786440408635135

曲 Published online: 08 Apr 2009.

Submit your article to this journal $₫$

Џ Article views: 2

Q View related articles $\square$ 
Solution of a Linear Differential Equation.

Hence, since

$$
\frac{\partial \mathrm{R}}{\mathrm{R}}=\frac{\partial \mathrm{T}}{\mathrm{T}}-\frac{1}{4} \mathrm{H}^{2} \frac{e^{2}}{m^{2}} \mathrm{~T}^{2}
$$

we have

$$
-13.9 \times 10^{-3}=23.47 \times 10^{-3}-\frac{1}{4}(17513)^{2} \cdot 10^{14} \cdot 1^{4},
$$

whence

$$
\mathrm{T}=2 \cdot 2 \times 10^{-12} \text { sec.*. }
$$

Indications seem to point to molecular and intermolecular effects of a highly complex nature, and until more definite information is available as to the structure of the molecule, it seems unlikely that a complete theory can be formulated.

In conclusion, we desire to express our obligation to Professor Taylor Jones, who suggested the research, for the valuable interest he has taken in the work, especially in the designing of the instrument employed in setting the specimen, the use of which has considerably enlarged the scope of the method.

Physics Laboratory,

University College of $\mathbf{N}$. Wales, Bangor, January 23rd, 1914.

LXXIV. The Sum of an Infinite Series as the Solution of a Linear Differential-Equation. By I. J. SchwatT †.

\section{Tro find}

$$
\mathrm{S}=\sum_{n=0}^{\infty} \frac{\boldsymbol{r}^{n}}{\prod_{m=1}^{6}(n+m)}, . . . . .
$$

Let $u_{n}$ denote the $(n+1)$ st term of the series, then

or

$$
\frac{u_{n}}{u_{n-1}}=r \frac{n}{n+6}
$$

and

$$
(n+6) u_{n}=r n u_{n-1}
$$

$$
\sum_{n=1}^{\infty}(n+6) u_{n}=r \sum_{n=1}^{\infty} n u_{n-1}=r \sum_{n=0}^{\infty}(n+1) u_{n}
$$

* J. J. Thomson, in his 'Corpuscular Theory of Matter,' gives $10^{-i} \mathrm{~cm}$. as the order of magnitude of the free path of a corpuscle, and $10^{7} \mathrm{~cm}$. per sec. as that of the velocity, whence $T=10^{-14}$ sec.

$\uparrow$ Communicated by the Author. 
Adding

$$
\left.(n+6) u_{n}\right]_{n=0}=\frac{1}{\underline{5}}
$$

to both sides of the last equation,

we have

$$
\sum_{n=0}^{\infty}(n+6) u_{n}=r \sum_{n=0}^{\infty}(n+1) u_{n}+\frac{1}{5 !} .
$$

But

therefore

$$
\sum_{n=0}^{\infty} u_{n}=\mathrm{S} \text { and } \sum_{n=0}^{\infty} n u_{n}=r \frac{d \mathrm{~S}}{d r}
$$

Now

$$
\begin{aligned}
& r \frac{d \mathrm{~S}}{d r}+6 \mathrm{~S}=r^{2} \frac{d \mathrm{~S}}{d r}+r \mathrm{~S}+\frac{1}{5 !}, \\
& r(1-r) \frac{d \mathrm{~S}}{d r}+(6-r) \mathrm{S}=\frac{1}{5 !} . . .
\end{aligned}
$$

Hence

$$
\begin{aligned}
\mathrm{S} & =\frac{e^{-\int \frac{6-r}{r(1-r)}} d r}{5 !} \int\left[\begin{array}{c}
e \int_{r(1-r)}^{6-r} d r \\
r(1-r)
\end{array}+\mathrm{C}\right] \\
& =\frac{1}{5 !} \frac{(1-r)^{5}}{r^{6}}\left[\int \frac{r^{5}}{(1-r)^{6}} d r+\mathrm{C}\right]
\end{aligned}
$$

$\begin{aligned} \frac{r^{3}}{(1-r)^{6}} l r & =\frac{r^{5}}{5(1-r)^{5}}-\frac{r^{4}}{4(1-r)^{4}}+\frac{r^{3}}{3(1-r)^{3}}-\frac{r^{2}}{2(1-r)^{2}}+\frac{r}{1-r}+\log (1-r) \\ & =\sum_{\kappa=1}^{5}(-1)^{\kappa-1} \frac{r^{\kappa}}{\kappa(1-r)^{k}}+\log (1-r) . \quad . . . . . . . . . .\end{aligned}$

Therefore

$$
\begin{aligned}
& =\frac{1}{5 !} \frac{(1-r)^{5}}{r^{6}} \log (1-r)+\frac{1}{5 ! r}\left[\frac{1}{5}-\frac{1-r}{4 r}+\frac{(1-r)^{2}}{3 r^{2}}-\frac{(1-r)^{3}}{2 r^{3}}+\frac{(1-r)^{4}}{r^{4}}\right] \\
& =\frac{1}{5 !} \frac{(1-r)^{5}}{r^{6}} \log (1-r)+\frac{1}{5 ! r}\left[\left(1-\frac{1}{r}\right)^{4}+\frac{1}{2}\left(1-\frac{1}{r}\right)^{3}\right. \\
& \left.+\frac{1}{3}\left(1-\frac{1}{r}\right)^{2}+\frac{1}{4}\left(1-\frac{1}{r}\right)+\frac{1}{5}\left(1-\frac{1}{r}\right)^{0}\right] \text {, } \\
& =\frac{1}{5 !} \frac{(1-r)^{5}}{r^{6}} \log (1-r)+\frac{1}{5 ! r} \sum_{k=1}^{5} \sum_{t=0}^{5-k}(-1)^{t} \frac{\left(\begin{array}{c}
5-t \\
t
\end{array}\right)}{\kappa r^{t}} \text {. } \\
& =\frac{1}{5 !} \frac{(1-r)^{5}}{r^{6}} \log (1-r)+\frac{1}{5 ! r}\left[\frac{137}{60}-\frac{77}{12 r}+\frac{47}{6 r^{2}}-\frac{9}{2 r^{3}}+\frac{1}{r^{4}}\right] \text {. }
\end{aligned}
$$

The same result might also be obtained by the following method. 
Solution of a Linear Differential Equation.

The given series (1) can be written

$$
\begin{aligned}
S & =\frac{1}{5 !} \sum_{\kappa=1}^{6}(-1)^{\kappa-1}\left(\begin{array}{l}
5 \\
\kappa-1
\end{array}\right) \sum_{n=0}^{\infty} \frac{r^{n}}{n+\kappa}, \ldots . . . \\
& =\frac{1}{5 !} \sum_{\kappa=1}^{6}(-1)^{\kappa-1}\left(\begin{array}{l}
5 \\
\kappa-1
\end{array}\right) \frac{1}{p^{\kappa}} \sum_{n=0}^{\infty} \frac{r^{n+\kappa}}{n+\kappa}, \ldots . . \\
& =\frac{1}{5 !} \sum_{k=1}^{6}(-1)^{\kappa-1}\left(\begin{array}{l}
5 \\
\kappa-1
\end{array}\right) \frac{1}{r^{\kappa}} \mathrm{S}_{k} . \quad . \quad . \quad . .
\end{aligned}
$$

Then

$$
\frac{d \mathrm{~S}_{\kappa}}{d r}=\sum_{n=0}^{\infty} r^{n+\kappa-1}=\frac{r^{\kappa-1}}{1-r} . \quad . \quad . \quad .
$$

Hence

$$
\mathrm{S}_{\kappa}=\int_{0}^{r} \frac{r^{\kappa-1}}{1-r} d r+\mathrm{C}_{\kappa} . \quad \text {. . . . . }
$$

From (12) follows :

$$
\begin{aligned}
& \mathrm{S}_{1}=\int_{0}^{r} \frac{d r}{1-r}=-\log (1-r) ; \\
& \mathrm{S}_{2}=\int_{0}^{r} \frac{r d r}{1-r}=-\log (1-r)-r ; \\
& \mathrm{S}_{3}=\int_{0}^{r} \frac{r^{2} d r}{1-r}=-\log (1-r)-r-\frac{r^{2}}{2} ; \\
& \mathrm{S}_{4}=\int_{0}^{r} \frac{r^{3} d r}{1-r}=-\log (1-r)-r-\frac{r^{2}}{2}-\frac{r^{3}}{3} ; \\
& \mathrm{S}_{5}=\int_{0}^{r} \frac{r^{4} d r}{1-r}=-\log (1-r)-r-\frac{r^{2}}{2}-\frac{r^{3}}{3}-\frac{r^{4}}{4} ; \\
& \mathrm{S}_{6}=\int_{0}^{r} \frac{r^{5} d r}{1-r}=-\log (1-r)-r-\frac{r^{2}}{2}-\frac{r^{3}}{3}-\frac{r^{4}}{4}-\frac{r^{5}}{5} .
\end{aligned}
$$

From (10) we obtain :

$$
\begin{aligned}
& S=\frac{1}{5} ! \sum_{\kappa=1}^{6}(-1)^{\kappa}\left(\begin{array}{l}
5 \\
\kappa-1
\end{array}\right) \frac{1}{r^{\kappa}} \log (1-r)+\frac{1}{5} \sum_{k=2}^{6}(-1)^{\kappa}\left(\begin{array}{l}
5 \\
\kappa-1
\end{array}\right) \frac{1}{r^{\kappa}} \sum_{t=1}^{\kappa-1} \frac{r^{t}}{t}
\end{aligned}
$$

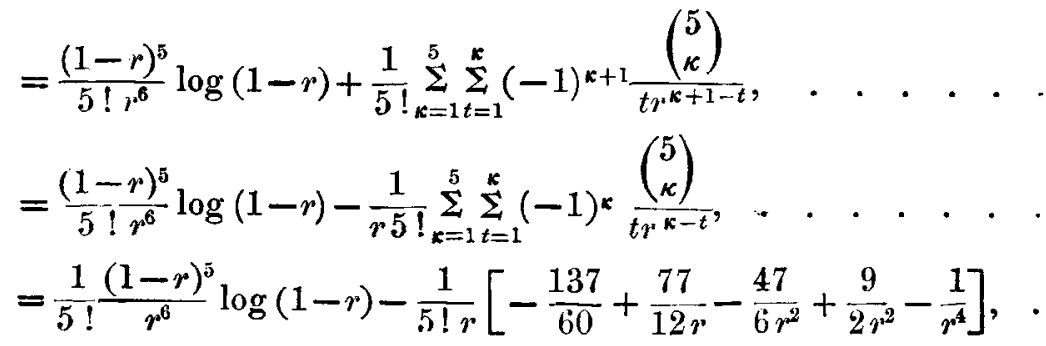

which is the same result as (7). 
662 Mr. W. H. Gibson on Influence of Volume Change

Again, the summation of the given series might be effected by a method similar to the above but without the use of integration.

The given series can be written

$$
\begin{aligned}
S & =\frac{1}{5 !} \sum_{\kappa=0}^{5}(-1)^{\kappa}\left(\begin{array}{l}
5 \\
\kappa
\end{array}\right) \sum_{n=0}^{\infty} \frac{r^{n}}{n+\kappa+1}, \quad . . . . . \\
& =\frac{1}{5 ! r^{2}} \sum_{n=0}^{5}(-1)^{\kappa}\left(\begin{array}{l}
5 \\
\kappa
\end{array}\right) \frac{1}{r^{*}} \sum_{n=0}^{\infty} \frac{r^{n+\kappa+1}}{n+\kappa+1} .
\end{aligned}
$$

Letting $n+\kappa+1=t$, we have

$$
\begin{aligned}
\mathrm{S} & =\frac{1}{5 ! r} \sum_{\kappa=0}^{5}(-1)^{\kappa}\left(\begin{array}{l}
5 \\
\kappa
\end{array}\right) \frac{1}{r^{\kappa}} \sum_{t=\kappa+1}^{\infty} \frac{r^{t}}{t}, \ldots . . . \\
& =\frac{1}{5 ! r} \sum_{\kappa=0}^{5}(-1)^{\kappa}\left(\begin{array}{l}
5 \\
\kappa
\end{array}\right) \frac{1}{r^{\kappa}}\left[\sum_{t=1}^{\infty} \frac{r^{t}}{t}-\sum_{t=1}^{\kappa} \frac{r^{t}}{t}\right],
\end{aligned}
$$

wherein

$$
\sum_{t=1}^{0} \frac{r^{t}}{t}=0
$$

Therefore

$$
\begin{aligned}
& \mathrm{S}=\frac{1}{5 ! r} \sum_{\kappa=0}^{5}(-1)^{\kappa}\left(\begin{array}{l}
5 \\
\kappa
\end{array}\right) \frac{1}{r^{\kappa}} \sum_{t=1}^{\infty} \frac{r^{t}}{t}-\frac{1}{5 ! r} \sum_{\kappa=1}^{5}(-1)^{\kappa}\left(\begin{array}{l}
5 \\
\kappa
\end{array}\right) \frac{1}{r^{\kappa}} \sum_{t=1}^{\kappa} \frac{r^{t}}{t} \\
& =-\frac{1}{5 ! r}\left(1-\frac{1}{r}\right)^{5} \log (1-r)-\frac{1}{5 ! r} \sum_{\kappa=1}^{5} \sum_{t=1}^{k}(-1)^{k} \frac{\left(\begin{array}{l}
5 \\
k
\end{array}\right)}{t r^{k-t}} \text {, . } \\
& =\frac{(1-r)^{5}}{5 ! r^{6}} \log (1-r)-\frac{1}{5 ! r} \sum_{\kappa=1}^{5} \sum_{t=1}^{k}(-1)^{\kappa} \frac{\left(\begin{array}{l}
5 \\
k
\end{array}\right)}{t r^{\kappa-t}} \text {. . . . . }
\end{aligned}
$$

Which is the same result as obtained in (14).

University of Pennsylvania, Philadelphia, U.S.A.

LXXV. The Influence of Volume Change on the Fluidity of Mixtures of Miscible Liquids. By WILLIAM HowIEson GIBSON *.

QEVERAL formulæ bave been used for the calculation of $D$ the fluidities or viscosities of mixtures of pairs of chemically indifferent, non-associated, completely miscible liquids from the fluidities or viscosities of the constituents,

* Communicated by Sir William Ramsay, K.C.B., F.R.S. 